\title{
DISCRETE LORENTZ SYMMETRIES IN GRAVITATIONAL FIELDS*
}

\author{
LESZEK M. SOKOŁOWSKI \\ Astronomical Observatory, Jagiellonian University \\ Orla 171, 30-244 Kraków, Poland \\ and \\ Copernicus Center for Interdisciplinary Studies, Kraków, Poland \\ lech.sokolowski@uj.edu.pl
}

(Received September 12, 2017)

\begin{abstract}
Belief in slight Lorentz symmetry violation at available energies is an imaginable part of belief in radically disparate physics near the Planck energy. As long as the effective field theory comprising new physics is formulated in flat spacetime, the description of hypothetical Lorentz symmetry violation within its formalism is consistent and tenable. If gravitation is introduced into the effective theory in the form of curved manifolds subject to Einstein field equations, the formalism of the symmetry violation encounters severe troubles. Either the dynamics of fundamental physical fields becomes inconsistent, or one is forced to accept existence of a large number of new species of fundamental matter beyond the Standard Model.
\end{abstract}

DOI:10.5506/APhysPolB.48.1947

In this talk addressed to nuclear physicists by a relativist, I present some concepts on conceivable impact of gravitational interactions on discrete symmetries in elementary particle physics. These are purely theoretical ideas since we live in this part of the universe where gravitation is very weak (what is very fortunate for us) and its influence on particle interactions is undetectable today. The concepts developed up to now concern the problems of whether Lorentz symmetries, both continuous and discrete, do hold in curved spacetimes and in what way they may be violated. Particularly interesting is the fate of discrete symmetries, C, P, T and CPT. I cannot, however, focus my attention on the discrete symmetries alone since they form a small sector of theory of Lorentz symmetries in the Standard Model

* Presented at the $2^{\text {nd }}$ Jagiellonian Symposium on Fundamental and Applied Subatomic Physics, Kraków, Poland, June 3-11, 2017. 
Extension including gravitation. This framework has arisen owing to increasing interest and progress in testing Lorentz invariance. Taking into account the importance of Lorentz invariance, there are two motivations for these investigations. Firstly, there is a significant increase of precision of experiments due to advances in technology. (To date, there is no compelling experimental evidence supporting any Lorentz symmetry violation.) Secondly, there are theoretical suggestions that Lorentz symmetry may not be exact at all interaction energies. The two leading theories of fundamental interactions, string theory (,theory of everything”) and quantum gravity (QG) suggest that the universe at energies close to the Planck energy, $E_{\mathrm{Pl}}=\left(\hbar c^{5} / G\right)^{1 / 2}=1,2 \times 10^{19} \mathrm{GeV}$, is completely different from the universe we live in. Both the theories remain in an early embryonic stage of their development and are not capable of providing concrete descriptions of physical processes, nevertheless they suggest some kinds of Lorentz symmetry violation (LSV). Here, I will focus on QG approach to Lorentz symmetries. Actually, LSV in quantum gravity is a conjecture and no model of QG does predict it uniquely. Therefore, there are two possibilities: (1) full QG does predict LSV at $E \approx E_{\mathrm{Pl}}$, then by continuity, at least small LSV occurs at low energies; (2) whereas exact QG is Lorentz invariant, it admits some tensor fields having nonzero vacuum expectation values. In the latter case, $\mathrm{VEV} \neq 0$ for these fields spontaneously break Lorentz symmetry of the lowenergy quantum state we live in, deceptively suggesting that exact QG also violates the symmetry. One then concludes that low-energy experiments cannot establish whether or not exact QG breaks Lorentz symmetry. In consequence, the available approach to the issue is based on a low-energy approximation to QG, called the Effective Field Theory (EFT). In practice, it takes the form of a non-quantum field theory with the Lagrangian of the Standard Model plus various low-energy additional terms involving all possible Lorentz symmetry violating operators coupled to physical fields (of the Model) and the gravitational sector; this kind of EFT is dubbed the Standard Model Extension (SME).

First, we explain what is meant by LSV in the framework of any EFT. Its definition asserts that Lorentz symmetry is violated if the underlying action integral for a physical field is not explicitly form-invariant under Lorentz transformations (continuous and/or discrete). This definition holds in flat Minkowski spacetime $\mathcal{M}_{4}$ (no gravity). Its meaning is shown in a simple example [1]. $\mathcal{M}_{4}$ has metric $\eta_{\mu \nu}$ and is endowed with an additional geometric structure in the form of a fixed constant symmetric tensor field $\zeta^{\mu \nu}$. In a given inertial frame $\mathrm{F}$, the field components have prescribed numerical values $\zeta^{\mu \nu}=$ const. Consider a real massless scalar field $\phi(x)$ additionally coupled to $\zeta^{\mu \nu}$, then its action integral reads

$$
S=\int_{D} \mathrm{~d}^{4} x\left[\eta^{\mu \nu} \frac{\partial \phi}{\partial x^{\mu}} \frac{\partial \phi}{\partial x^{\nu}}+\zeta^{\mu \nu} \frac{\partial \phi}{\partial x^{\mu}} \frac{\partial \phi}{\partial x^{\nu}}\right]
$$


both $S$ and integrand terms are scalars. Let $L$ be a Lorentz transformation from $\mathrm{F}$ to another inertial frame, $y=L x$, then $L$ maps the integration domain $D$ onto $D^{\prime}=L^{-1}(D)$. Since $\phi$ is a scalar, $\phi(x)=\phi\left(L^{-1} y\right) \equiv \phi^{\prime}(y)$, the action is numerically unaltered

$$
S=\int_{D^{\prime}} \mathrm{d}^{4} y\left[\eta^{\mu \nu} \frac{\partial \phi^{\prime}}{\partial y^{\mu}} \frac{\partial \phi^{\prime}}{\partial y^{\nu}}+\zeta^{\prime \mu \nu} \frac{\partial \phi^{\prime}}{\partial y^{\mu}} \frac{\partial \phi^{\prime}}{\partial y^{\nu}}\right] .
$$

Here, $\eta^{\mu \nu}=\eta^{\prime \mu \nu}=\operatorname{diag}[1,-1,-1,-1]$ has this form in all inertial frames (is form-invariant), whereas

$$
\zeta^{\prime \mu \nu}=L_{\alpha}^{\mu} L^{\nu} \zeta^{\alpha \beta} \neq \zeta^{\mu \nu}
$$

is not form-invariant since these are two different matrices. One infers that the term $\zeta^{\mu \nu} \partial_{\mu} \phi \partial_{\nu} \phi$ in $S$ causes LSV because it is not form-invariant under $L$ due to presence of a non-dynamical operator (tensor) $\zeta^{\mu \nu}$ which is frame-dependent. I stress that the form-dependence arises since $\zeta^{\mu \nu}$ is nondynamical and expresses an additional fixed geometrical structure of flat spacetime.

In some inertial frames, $\zeta^{\mu \nu}$ takes on particularly simple form and/or is small implying that LSV is small there. In many models, the Lorentz symmetry violating tensors, such as $\zeta^{\mu \nu}$, may be reduced to a vector field determining a distinguished direction in the spacetime. There is one wellknown example of a distinguished vector field defining a preferred ("concordant") reference frame: this is a timelike vector determining the rest frame of the black-body cosmic background radiation being a remnant of hot plasma epoch of evolution of the early universe. However, this field and the preferred frame are related to the global evolving the Robertson-Walker geometry of the universe, thus it is relevant on cosmological scales, whereas all the Lorentz symmetry violating operators (tensor fields) are assumed to work on microscopic scales.

Being a tensor, $\zeta^{\mu \nu}$ is always different from zero and thus it generates LSV in all inertial frames. The existence of preferred frames (directions in spacetime) is not necessary and occurs only in simple models of SME. If there are two Lorentz invariance violating tensors, $\zeta^{\mu \nu}$ and $\chi^{\mu \nu}$, then there are no preferred frames.

As long as one considers EFT in flat spacetime, the concept of LSV by specific fields coupled to known physical fields of the Standard Model is theoretically well-defined [1-4]. Conceptual troubles arise when gravitational interactions are included, particularly for discrete symmetries. 
In flat spacetime, the space inversion and time reversal are significant since one uses inertial frames and Cartesian coordinates in them, which reveal geometrical properties of the spacetime. Time coordinate measures proper time elapsing in a given frame and spatial coordinates directly measure distances in space between simultaneous events. Inversion of time and space has thus a direct geometrical meaning and it is of crucial importance whether physical processes respect these geometrical symmetries. This is, however, specific to flat spacetime with its metric affine structure. Gravitation radically changes this picture. According to general relativity (GR), gravitational interactions make the very spacetime a dynamical material object, though quite specific; each gravitational field forms its own curved spacetime. And a general curved spacetime has no symmetries, curvature annihilates the linear affine structure. There are no priviledged reference frames, neither inertial nor any other. Following fundamental concepts of differential geometry, all possible reference frames are on equal footing and accordingly spacetime coordinates applied in each of them are arbitrary and are devoid of any geometrical interpretation. They are merely four numbers labelling in a continuous way spacetime points (events). Something similar occurs to the notion of space. In flat spacetime, each inertial frame determines a slicing of the spacetime by a collection of spaces defined as 3 -dimensional sets of events simultaneous in this frame. In a curved spacetime, the time coordinate of a given coordinate system also defines the space as a hypersurface of events having the same value of this coordinate, but taking into account the arbitrariness of choice of time coordinate, the corresponding slicing of the spacetime by spaces is to a large extent arbitrary. I emphasize that in gravitational field a time coordinate is distinct from the proper time measured by physical clocks (and each clock has its own proper time equal to the length of its worldline, so that there is no common proper time) and spatial coordinates are distinct from geometrically defined spatial distances.

In a generic spacetime, its curvature evolves in space and time and has no symmetries. If there is no translational invariance in a timelike direction (,in time"), there is no way of defining a time reversal $\mathrm{T}$ and if there is no spacelike direction revealing translational invariance of space, then no concept of space inversion $\mathrm{P}$ may exist.

What about special spacetimes possessing some symmetries: e.g. Schwarzschild's (time translation and rotational symmetry about the space centre) or the Robertson-Walker (space is homogeneous and isotropic)? In principle, one might attempt to define some discrete symmetries in them, but these would only fit this particular spacetime. And these would be of very little usefulness. In fact, when in future the experiments will be sufficiently precise, we will detect in particle interactions influence of the gravitational field with low symmetry, being a nonlinear composition of the Sun's, Earth's and cosmological gravity. 
In a curved spacetime, there are no global symmetries, yet the local Lorentz invariance holds: at each point, there is the whole set of local Lorentz (inertial) frames and none of them is preferred and there are no distinguished directions at this point.

As concerns continuous LSV, one takes gravitational effects into account in a simple way by applying the Minimal Coupling Principle ((MCP), sometimes called "minimal substitution rule": one takes the Lagrangian of the EFT under consideration expressed in Minkowski spacetime, assumes that all the tensors present in it, which have initially been supposed to be Lorentz covariant, are actually general covariant (under arbitrary nonlinear coordinate transformations) and replaces Minkowski metric $\eta_{\mu \nu}$ by a generic metric $g_{\mu \nu}$ and finally, replaces partial derivatives by covariant ones. In this way, the Lagrangian becomes generally covariant and it is expected that the terms violating continuous Lorentz symmetry in flat spacetime will do it in presence of curvature [3] (that is, while having the generally covariant form). The violation now means that at each spacetime point, there appears an additional geometric structure (such as a preferred direction), independent of the metric, which may distinguish one (or a class of) local Lorentz frame.

As motivated above, in gravitational fields, the discrete $\mathrm{P}$ and $\mathrm{T}$ symmetries have no sense. Yet in quantum field theory (QFT) in flat spacetime, the CPT symmetry plays so fundamental role expressed in the CPT theorem, that it is legitimate to ask of what occurs to it in curved spacetimes. There appear two problems. First, there are severe conceptual troubles with formulating a genuine quantum theory in a variable gravitational field, particularly in presence of black holes. In consequence, the issue of a gravitational analogue of the CPT theorem in curved spacetimes is not urgent. Second, it is obvious from the above discussion that establishing a satisfactory definition of CPT symmetry in presence of curvature is hard if possible at all. Nonetheless, the fate of CPT symmetry in gravitation, even weak, should not be neglected. Recall that in lack of a genuine QFT, one employs a quasi-classical EFT. It seems plausible that some analogue of CPT symmetry in GR should exist, though a precise definition is missing today. And circumventing the issue of its definition, one may conjecture that this "quasi-CPT" is violated in a way determined by again applying MCP [3]: in gravitational field CPT symmetry is violated by the covariant form of those terms in the action which violate exact CPT symmetry in flat spacetime.

However, one may challenge this simple conjecture because discrete symmetries, such as CPT, are global ones, whereas MCP assumes local, i.e. continuous transition from flat to curved spacetime. This subtle issue definitely deserves a detailed study. 
Let us assume that application of MCP is sufficient at least in the case of continuous LSV and seek its consequences. It turns out that continuous LSV gives rise to the Einstein Equivalence Principle violation and other severe troubles [1]; here, I will discuss consistency of the approach to symmetry violation as such. In flat spacetime, LSV is achieved by introduction of a constant tensor field $\zeta^{\mu_{1} \ldots \mu_{k}}$ with $k$ indices $\left(\zeta^{\mu \nu}\right.$ in the example with the massless scalar field) which is directly coupled to physical matter fields. In GR, the constant $\zeta$ must be replaced by a point-dependent $\zeta^{\mu_{1} \ldots \mu_{k}}(x)$ since a constant tensor in one frame becomes point dependent under any nonlinear coordinate transformation and this dependence must be established in the initial frame. A priori there are two possibilities: either $(1) \zeta(x)$ is a fixed non-dynamical field or $(2) \zeta(x)$ is a new (unknown) physical field with its own equations of motion, then it is determined in each frame as a solution satisfying given initial data.

It seems obvious that $\zeta(x)$ should be a fixed field without its own dynamics (and form "an absolute element of the theory"), but it usually leads to inconsistencies. In most models, tensor $\zeta$ is decomposed into a product of one or more vector fields. It is natural to assume that a constant tensor in flat spacetime should be replaced by a covariantly constant tensor in a curved spacetime, $\partial_{\alpha} \zeta^{\mu_{1} \ldots \mu_{k}}=0 \Rightarrow \nabla_{\alpha} \zeta^{\mu_{1} \ldots \mu_{k}}=0$, and the constituent vector fields also must be covariantly constant. This is, however, impossible: contrary to our intuition, which has been formed in Euclidean space, in most curved spacetimes covariantly constant vector (and tensor) fields do not exist; for example, there is no covariantly constant vector field on the sphere. Spacetimes admitting covariantly constant vector fields are exceptional, e.g. plane gravitational waves. Instead, the tensor $\zeta(x)$ must be carefully adjusted and there is no clear rule of how to do it uniquely. Suppose that the spacetime metric depends on some parameters and for their particular values the spacetime becomes flat (for instance, the mass is zero in Schwarzschild metric). Adjust (usually non-uniquely) the tensor $\zeta(x)$ dependent on these parameters so that $\zeta=$ const in the flat spacetime limit. Now, take the above example of a scalar massless field $\phi$ coupled to the adjusted $\zeta^{\mu \nu}(x)$; it may be shown that $\zeta^{\mu \nu}$ generates continuous LSV and there is no hypothetical CPT violation [3, 4]. Now, the action functional for $\phi$ includes the GR Lagrangian and reads [1]

$$
S=\int_{D} \mathrm{~d}^{4} x \sqrt{-g}\left[\frac{1}{16 \pi G} R+\frac{1}{2}\left(g^{\mu \nu}+\zeta^{\mu \nu}(x)\right) \frac{\partial \phi}{\partial x^{\mu}} \frac{\partial \phi}{\partial x^{\nu}}\right] .
$$

It gives rise to the Lagrange equations of motion for $\phi$,

$$
\square \phi+\nabla_{\mu}\left(\zeta^{\mu \nu} \frac{\partial \phi}{\partial x^{\nu}}\right)=0
$$


and the Einstein field equations

$$
\begin{aligned}
G_{\alpha \beta} & =8 \pi G T_{\alpha \beta} \equiv \\
& \equiv 8 \pi G\left[\frac{\partial \phi}{\partial x^{\alpha}} \frac{\partial \phi}{\partial x^{\beta}}-\frac{1}{2}\left(g^{\mu \nu}+\zeta^{\mu \nu}(x)\right) \frac{\partial \phi}{\partial x^{\mu}} \frac{\partial \phi}{\partial x^{\nu}} g_{\alpha \beta}\right]
\end{aligned}
$$

The Einstein tensor satisfies the Bianchi identity $\nabla_{\beta} G_{\alpha}^{\beta} \equiv 0$ which implies four equations for the energy-momentum tensor $\nabla_{\beta} T_{\alpha}^{\beta}=0$. For $T_{\alpha \beta}$ given in (5), this yields

$$
\nabla_{\beta} T_{\alpha}^{\beta}=-\frac{\partial \phi}{\partial x^{\alpha}} \nabla_{\mu}\left(\zeta^{\mu \nu} \frac{\partial \phi}{\partial x^{\nu}}\right)-\frac{1}{2} \nabla_{\alpha}\left(\zeta^{\mu \nu} \frac{\partial \phi}{\partial x^{\mu}} \frac{\partial \phi}{\partial x^{\nu}}\right)
$$

and this divergence cannot vanish for the adjusted $\zeta^{\mu \nu}$ and for each solution $\phi$ of (4). This is clearly inconsistent (actually contradictory). The divergence might be zero only for very special solutions $\phi$, for instance $\phi=0$. Furthermore, Eqs. (5) show that $\zeta^{\mu \nu}$ is a part of the source determining the metric, contrary to the assumption that $g_{\mu \nu}$ and $\zeta^{\mu \nu}$ are independent of each other.

To restore consistency one concludes:

in the presence of gravitation, the Lorentz symmetry violating tensor $\zeta$ must be dynamical and represent a new kind of matter, beyond the Standard Model.

This is an unexpected prediction of the conjecture of LSV in low energy EFT such as the Standard Model Extension. The same holds for the terms violating the analogue of CPT symmetry. As a relativist, I doubt whether postulating existence of so many (tens if not hundreds [4, 5]) new species of fundamental matter is reasonable.

This work was supported by a grant from the John Templeton Foundation.

\section{REFERENCES}

[1] D. Mattingly, Living Rev. Relativ. 8, 5 (2005).

[2] S. Liberati, Class. Quantum Grav. 30, 133001 (2013).

[3] V.A. Kostelecky, Phys. Rev. D 69, 105009 (2004) [arXiv:hep-th/0312310].

[4] V.A. Kostelecky, N. Russell, Rev. Mod. Phys. 83, 11 (2011) [arXiv:0801.0287 [hep-ph]].

[5] V.A. Kostelecky, arXiv:1610.09284 [hep-ph]. 